\title{
Induction chemotherapy followed by concurrent chemoradiation and nimotuzumab for locoregionally advanced nasopharyngeal carcinoma: preliminary results from a phase II clinical trial
}

\author{
Jian-feng Huang 1,2,*, Fu-zheng Zhang ${ }^{2, *}$, Qin-zhou Zou ${ }^{2}$, Le-yuan Zhou ${ }^{2}$, Bo Yang ${ }^{2}$,

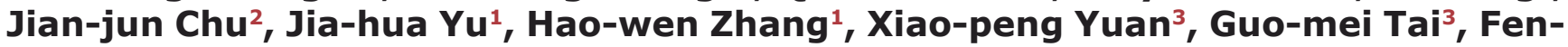 \\ ju Liu ${ }^{1}$ and C-M Charlie $\mathrm{Ma}^{4}$ \\ ${ }^{1}$ Department of Radiobiology, School of Radiation Medicine and Protection and Jiangsu Provincial Key Laboratory of Radiation \\ Medicine and Protection, Medical College of Soochow University, Collaborative Innovation Center of Radiation Medicine of \\ Jiangsu Higher Education Institutions and School for Radiological and Interdisciplinary Sciences (RAD-X), Soochow University, \\ Suzhou, China \\ 2 Department of Radiation Oncology, Affiliated Hospital of Jiangnan University, Wuxi, China \\ ${ }^{3}$ Department of Radiation Oncology, Nantong Tumor Hospital, Affiliated Tumor Hospital of Nantong University, Nantong, \\ China \\ ${ }^{4}$ Department of Radiation Oncology, Fox Chase Cancer Center, Philadelphia, PA, USA \\ * These authors have contributed equally to this work \\ Correspondence to: Fen-ju Liu, email: fangsh@suda.edu.cn \\ Keywords: nasopharyngeal carcinoma, nimotuzumab, chemoradiotherapy, induction chemotherapy \\ Received: October 13,2016 Accepted: November 24, $2016 \quad$ Published: December 10, 2016
}

ABSTRACT

Overexpression of epidermal growth factor receptor can be found in more than $\mathbf{8 0} \%$ of patients with locoregionally advanced nasopharyngeal carcinoma and is associated with shorter survival. In this work, we evaluated the feasibility of adding nimotuzumab to chemoradiation in locoregionally advanced nasopharyngeal carcinoma. Twenty-three patients with clinically staged T3-4 or any node-positive disease were enrolled. They were scheduled to receive one cycle of induction chemotherapy followed by intensity-modulated radiotherapy, weekly administration of nimotuzumab and concurrent chemotherapy. Results showed that all patients received a full course of radiotherapy, 19(82.6\%)patients completed the scheduled neoadjuvant and concurrent chemotherapy, and $22(95.7 \%)$ patients received $\geq 6$ weeks of nimotuzumab. During the period of concurrent chemoradiation and nimotuzumab, grade 3-4 toxicities occurred in $14(60.9 \%)$ patients: $8(34.8 \%)$ had grade 3-4 oral mucositis, $6(26.1 \%)$ had grade 3 neutropenia, and $1(4.3 \%)$ had grade 3 dermatitis. No acne-like rash was observed. With a median follow-up of 24.1 months, the 2-year progression-free survival and overall survival were $83.5 \%$ and $95.0 \%$, respectively. In conclusion, concurrent administration of chemoradiation and nimotuzumab was well-tolerated with good compliance. Preliminary clinical outcome data appear encouraging with favorable normal tissue toxicity results comparing with historical data of concurrent chemoradiation plus cetuximab.

\section{INTRODUCTION}

Concurrent chemoradiation(CCRT) is the standard combinational treatment modality for locoregionally advanced nasopharyngeal carcinoma(LA NPC). With the addition of platinum-based concurrent chemotherapy, a significant survival benefit has been achieved compared with radiotherapy alone $[1,2]$.However, up to $30 \%$ of patients still die of distant metastasis, while 10\%-20\% of patients will develop local and regional recurrences [3-5].The optimum sequence and combination of chemoradiotherapy for the treatment of LA NPC remains controversial. Newer treatment modalities, including different sequences and combinations of chemoradiotherapy are explored. 
Table 1: Baseline characteristics of patients

\begin{tabular}{|c|c|}
\hline Characteristic & Number of patients \\
\hline Total & 23 \\
\hline \multicolumn{2}{|l|}{ Gender } \\
\hline Male & 18 \\
\hline Female & 5 \\
\hline \multicolumn{2}{|l|}{ Age,years } \\
\hline Median & 53 \\
\hline Range & $23-73$ \\
\hline \multicolumn{2}{|c|}{ WHO histologic type } \\
\hline II & 18 \\
\hline III & 5 \\
\hline \multicolumn{2}{|c|}{ Clinical T category } \\
\hline $\mathrm{T} 1$ & 1 \\
\hline $\mathrm{T} 2$ & 7 \\
\hline $\mathrm{T} 3$ & 13 \\
\hline $\mathrm{T} 4$ & 2 \\
\hline \multicolumn{2}{|l|}{ Clinical $N$ category } \\
\hline N0 & 1 \\
\hline N1 & 5 \\
\hline N2 & 11 \\
\hline N3 & 6 \\
\hline \multicolumn{2}{|l|}{ UICC stage } \\
\hline II & 3 \\
\hline III & 12 \\
\hline IVa & 2 \\
\hline $\mathrm{IVb}$ & 6 \\
\hline
\end{tabular}

Abbreviations: WHO, World Health Organization; UICC, Union for International Cancer Control; T, tumor; N, lymph node

On the other hand, overexpression of EGFR can be found in more than $80 \%$ of patients with LA NPC [6]. High EGFR expression was associated with radiotherapy and chemotherapy resistance, and increased risks of locoregional recurrence, distant metastasis, and poor prognosis $[7,8]$. Cetuximab, the most commonly used anti-EGFR antibody, has received considerable attention and achieved encouraging progress for the treatment of head and neck squamous cell carcinoma(HNSCC) [911]. The schedule of CCRT with cetuximab in LA NPC [12-15] has also demonstrated promising preliminary results. However, the incidence of acne-like rash and radiotherapy-related acute skin and mucosal toxic effects was significantly increased, thus its clinical application was greatly limited.

Distinct from cetuximab, nimotuzumab is a humanized EGFR monoclonal antibody with a unique safety profile [16]. However, there is little research focusing on the use of CCRT in combination with nimotuzumab in LA NPC. In the phase II single-arm trial presented here, we adopted a combinational treatment modality of induction chemotherapy, followed by concurrent chemoradiation and nimotuzumab for the treatment of LA NPC (Figure 1). The main purpose of the study is to evaluate the safety and treatment compliance of this treatment regimen. Here, we present the preliminary results of our study.

\section{RESULTS}

\section{Patients and treatment compliance}

From November 2011 to April 2016, 23 patients with a median age of 53 years (range, 23-73 years) were recruited into the study, baseline characteristics are listed in Table 1. All patients received one cycle of induction chemotherapy and a full course of intensity-modulated radiotherapy (IMRT) (Table 2). IMRT was interrupted in two patients who experienced severe side effects of grade 4 stomatitis $(n=1)$ and grade 3 dermatitis $(n=$ 1). Nineteen $(82.6 \%)$ patients completed the scheduled concurrent chemotherapy. In terms of compliance to nimotuzumab, $22(95.7 \%)$ patients received $\geq 6$ weeks of nimotuzumab, $19(82.6 \%)$ patients received 8 weeks of nimotuzumab. Nimotuzumab was discontinued in 4 patients due to refusal by patient $(n=3)$ and anaphylaxis 
Table 2: Treatment compliance

\begin{tabular}{|l|c|}
\hline \multicolumn{1}{|c|}{ Treatment compliance } & Number of patients(\%) \\
\hline Induction chemotherapy & $23(100)$ \\
\hline received one cycle of induction chemotherapy & \\
\hline Concurrent chemoradiation & $23(100)$ \\
\hline Radiotherapy & $2(8.7)$ \\
\hline received total dose of radiotherapy & $0(0)$ \\
\hline radiotherapy interruptions & \\
\hline radiotherapy discontinuation & $19(82.6)$ \\
\hline Chemotherapy & $4(17.4)$ \\
\hline received one cycle of concurrent chemotherapy & \\
\hline did not receive concurrent chemotherapy & $19(82.6)$ \\
\hline Nimotuzumab & $4(17.4)$ \\
\hline received eigtht weeks of nimotuzumab & $1(4.3)$ \\
\hline did not receive eigtht weeks of nimotuzumab & $2(8.7)$ \\
\hline received 7 weeks of nimotuzumab & $1(4.3)$ \\
\hline received 6 weeks of nimotuzumab & \\
\hline received 1 weeks of nimotuzumab & $3(13.0)$ \\
\hline Adjuvant chemotherapy & $20(87.0)$ \\
\hline not scheduled to receive adjuvant chemotherapy & $18(78.4)$ \\
\hline scheduled to receive adjuvant chemotherapy & $1(4.3)$ \\
\hline received 4 cycles of adjuvant chemotherapy & $1(4.3)$ \\
\hline received 3 cycles of adjuvant chemotherapy & \\
\hline received 2 cycles of adjuvant chemotherapy & \\
\hline
\end{tabular}

Table 3: Adverse effects from concurrent chemoradiation and nimotuzumab

\begin{tabular}{|c|c|c|c|}
\hline \multirow{2}{*}{ Adverse effects } & \multicolumn{3}{|c|}{ Number of patients(\%) } \\
\cline { 2 - 4 } & Grade 1-2 & Grade 3 & Grade 4 \\
\hline Neutropenia & $11(47.8)$ & $6(26.1)$ & $0(0)$ \\
\hline Thrombocytopenia & $3(13.0)$ & $0(0)$ & $0(0)$ \\
\hline Anemia & $2(8.7)$ & $1(4.3)$ & $0(0)$ \\
\hline Oral mucositis & $15(65.2)$ & $7(30.5)$ & $0(0)$ \\
\hline Xerostomia & $20(87.0)$ & $3(13.0)$ & $0(0)$ \\
\hline Dermatitis & $19(82.6)$ & $1(4.3)$ & $0(0)$ \\
\hline
\end{tabular}

Table 4: Grade 3-4 adverse effects in 19 patients who actually received concurrent chemoradiation and nimotuzumab

\begin{tabular}{|c|c|}
\hline Grade 3-4 adverse effects & Number of patients(\%) \\
\hline Neutropenia & $5(26.3)$ \\
\hline Anemia & $1(5.3)$ \\
\hline Oral mucositis & $7(36.8)$ \\
\hline Xerostomia & $2(10.5)$ \\
\hline Dermatitis & $1(5.3)$ \\
\hline
\end{tabular}

$(n=1)$. Adjuvant chemotherapy was administered in $20(87 \%)$ patients: 18 received four cycles of adjuvant chemotherapy, 1 received three cycles, and another received two cycles.

\section{Toxicity}

No grade 3-4 toxicities occurred in the neoadjuvant setting. Adverse events from concurrent chemoradiation and nimotuzumab were listed in Table 3. The most common side effects were oral mucositis and neutropenia. Grade 3 or higher acute toxicities occurred in 14 (60.9\%) of 23 patients: oral mucositis was recorded as grade 3 in 7 (30.5\%) patients and grade 4 in $1(4.3 \%)$ patient; $6(26.1 \%)$ patients suffered with grade 3 neutropenia, 3(13\%) with grade 3 xerostomia, and $1(4.3 \%)$ with grade 3 anemia. One case of grade 3 dermatitis was observed within the radiation field. Of the 19 patients who actually received concurrent chemotherapy, grade 3-4 oral mucositis, 
Table 5: Response to treament (3months after radiotherapy)

\begin{tabular}{|c|c|c|c|c|c|}
\hline \multirow{2}{*}{ Site } & \multicolumn{5}{|c|}{ Number of patients(\%) } \\
\cline { 2 - 6 } & CR & PR & SD & PD & CR+PR \\
\hline Nasopharynx $(\mathrm{n}=23)$ & $21(91.3 \%)$ & $2(8.7 \%)$ & $0(0)$ & $0(0)$ & $23(100 \%)$ \\
\hline Regional lymph nodes $(\mathrm{n}=22)$ & $21(95.5 \%)$ & $1(4.5 \%)$ & $0(0)$ & $0(0)$ & $22(100 \%)$ \\
\hline
\end{tabular}

Abbreviations: CR, Complete response; PR, Partial response; SD, Stable disease; PD, Progress disease

Table 6: Comparision of adverse effects with previously published studies treated with CCRT+cetuximab

\begin{tabular}{|l|c|c|c|c|c|}
\hline & \multicolumn{5}{|c|}{ Study } \\
\hline & Feng et al. & Ma et al. & Niu et al. & He et al. & Our series \\
\hline Number of patients & 28 & 30 & 33 & 21 & 23 \\
\hline Year & 2014 & 2012 & 2013 & 2013 & 2016 \\
\hline All grades rash(\%) & 85.8 & 93 & 93.9 & 100 & 0 \\
\hline Grade 3/4 rash(\%) & 17.9 & 10 & 39.4 & 19.1 & 0 \\
\hline Grade 3/4 mucositis(\%) & 71.4 & 87 & 84.9 & 66.7 & 36.8 \\
\hline Grade 3/4 dermatitis(\%) & 25 & 20 & 18.2 & 23.8 & 5.3 \\
\hline
\end{tabular}

Abbreviations: CCRT, Concurrent chemoradiation

dermatitis and neutropenia occurred in 7(36.8\%), 1(5.3\%) and $5(26.3 \%)$ patients, respectively(Table 4$)$. With respect to the toxicities of nimotuzumab, only 1 patient suffered anaphylaxis, no acne-like rash was found in any patients. In the adjuvant setting, the incidence rate of grade 3-4 acute toxicities in our cohort was 25\% (5/20): 4(20\%) patients with grade 4 neutropenia, and $1(5 \%)$ with grade 3 neutropenia.

Xerostomia and hearing impairment were the most common late toxicities, and the degree decreased over time during follow-up. One patient experienced nasopharyngeal ulcer and bleeding, and recovered after symptomatic treatment. There were no cases of radiation-induced cranial nerve palsy, temporal lobe necrosis, osteonecrosis, myelopathy, or pituitary damage. There were no treatmentrelated deaths.

\section{Treatment response and survival}

At the first 3-month follow-up after the completion of radiation, of the 23 patients, complete response (CR) and partial response (PR) of nasopharynx were achieved in $21(91.3 \%)$ and $2(8.7 \%)$ patients, respectively. Of the
22 patients with regional nodal involvement at diagnosis, 21 showed CR and 1 PR(Table 5). The residual tumor gradually disappeared or became less obvious at the following time.

At a median follow-up of 24.1months [range, 5.0 58.2months], the estimated 2-year progressionfree survival(PFS) was $83.5 \%$ (95\% CI56.1\% to $94.5 \%$ ) (Figure 2), and the estimated 2-year overall survival(OS) was $95.0 \%$ (95\% CI $69.5 \%$ to $99.3 \%$ )(Figure 3). At the last follow-up, a total of 5 patients failed with distant metastasis: 3 patients with bone metastases only, 1 with lung and bone metastases and 1 with liver and bone involvement, and three of them had died. Locoregional recurrence was not observed.

\section{DISCUSSION}

The main objective of this phase II study is to determine the toxic effects and compliance of adding nimotuzumab to chemoradiation in LA NPC. Initial results show that this combinational treatment modality is feasible, with good compliance and acceptable toxicity. All patients received a full course of radiotherapy,

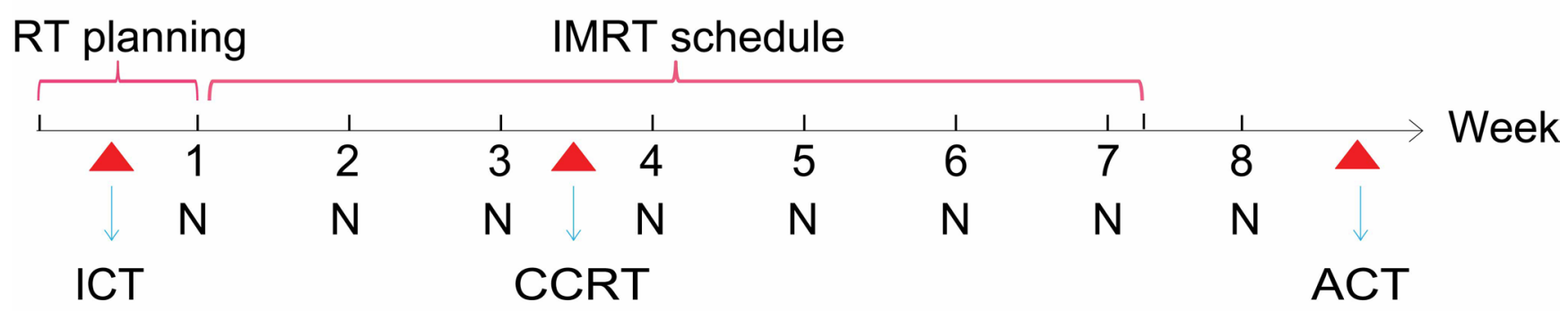

Figure 1: Multidisciplinary management of LA NPC in our study. Abbreviations: LA NPC, Locoregionally advanced nasopharyngeal carcinoma; RT, Radiotherapy; IMRT, Intensity-modulated radiotherapy; ICT, Induction chemotherapy; CCRT, Concurrent chemotherapy; ACT, Adjuvant chemotherapy; N, Nimotuzumab 
$19(82.6 \%)$ patients completed the scheduled neoadjuvant and concurrent chemotherapy, and $22(95.7 \%)$ patients received $\geq 6$ weeks of nimotuzumab. No acne-like rash was observed. The incidence rate of grade 3-4 acute toxicities from concurrent chemoradiation and nimotuzumab in our cohort was $60.9 \%$.

As with other studies of CCRT, radiation-induced oral mucositis was the most common acute side-effect. In our study, Grade 3-4 oral mucositis occurred in 36.8\% of patients who actually received CCRT+nimotuzumab. Compared with other studies of CCRT regimens, in which Grade 3-4 oral mucositis was reported between 29\% and $52.4 \%$ [5, 17-19], the incorporation of nimotuzumab in our cohort was well-tolerated, without aggravation of oral mucositis. We also noted that $6(26.1 \%)$ patients suffered with grade 3 neutropenia, which was higher than that reported by a previous study [19]. Gastrointestinal side-effects, including anorexia, nausea and vomiting, were relatively mild in this study. We attribute this to the characteristic feature of nedaplatin, a derivative of cisplatin with less gastrointestinal toxicity but greater hematologic toxicity.

With the optimized local treatment by IMRT, locoregional recurrence rates have been markedly reduced [3-5]. In our study, no locoregional recurrence was observed. The main cause for failure in NPC is now distant metastases, especially in these with advanced disease at the primary site or regional lymph nodes. In the present study, $65.2 \%$ and $73.9 \%$ of patients developed T3-T4 and N2-N3 disease, respectively, and 5 patients developed one or more systemic diseases. However, our results should be regarded as preliminary because of the relatively small sample size and short follow-up.

At present, concurrent chemotherapy during the course of radiotherapy should be considered the standard of care for LA NPC. In addition to conventional 3 -weekly $(100 \mathrm{mg} / \mathrm{m} 2)$ or weekly $(30-40 \mathrm{mg} / \mathrm{m} 2)$ schedule of cisplatin, other concurrent agents and regimes combined with radiotherapy have been explored. In a retrospective study by $\mathrm{Xu}$ and colleagues [20], the regime of paclitaxel plus nedaplatin was administered, comparable survival to those of the cisplatin-based

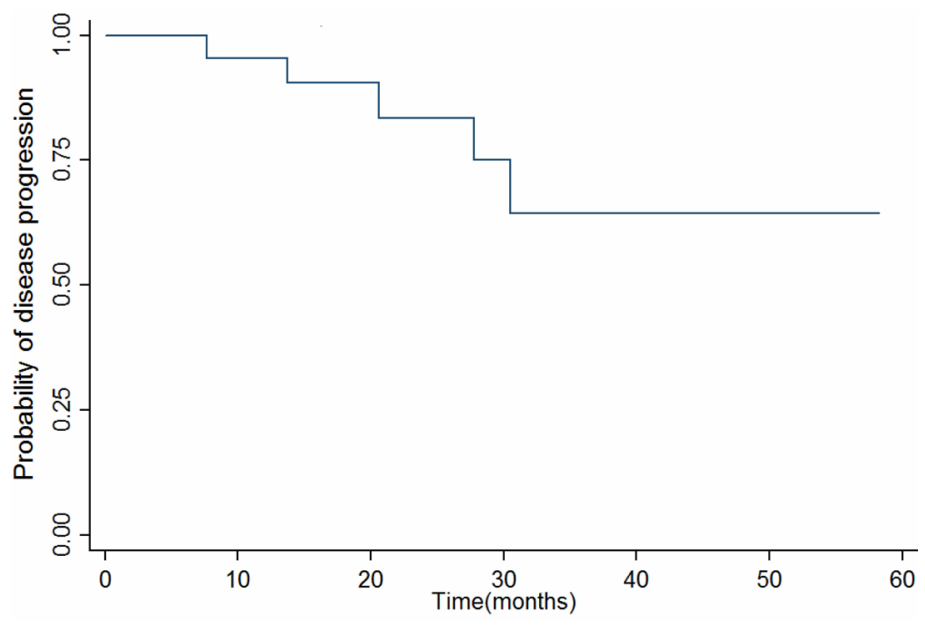

Figure 2: Kaplan-Meier survival estimate of PFS. Abbreviations: PFS, Progression-free survival.

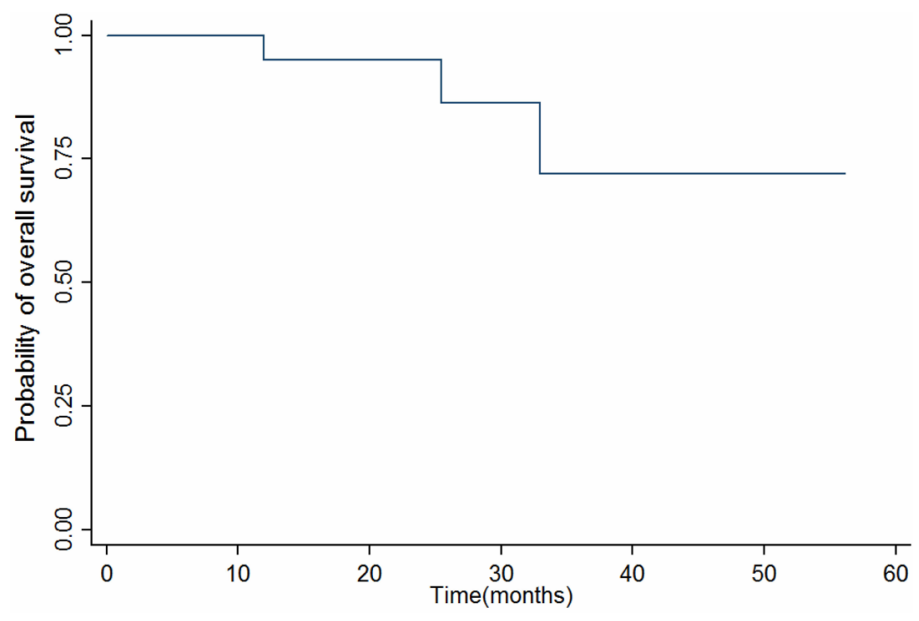

Figure 3: Kaplan-Meier survival estimate of OS. Abbreviations: OS, Overall survival. 
regimens was found. Besides, considering that many patients with LA NPC demonstrate excessive expression of EGFR, and EGFR overexpression is associated with increased risks of distant metastasis and shorter survival, the strategy of EGFR inhibition seems attractive and has demonstrated encouraging clinical outcomes [21, 22]. However, evidence from published data is still insufficient to warrant changing the standard of care from chemoradiation to EGFR antagonists plus radiotherapy. In this preliminary study, we incorporated nimotuzumab into chemoradiation, the couplet regimen of docetaxel $(75 \mathrm{mg} /$ $\mathrm{m} 2)$ plus nedaplatin $(80 \mathrm{mg} / \mathrm{m} 2)$ and weekly schedule of nimotuzumab were administered as concurrent agents. Concurrent chemotherapy was administered at intervals of 4 weeks after induction chemotherapy, which almost at the 4th week of radiotherapy. And then, during the next 3 weeks of radiotherapy, weekly nimotuzumab alone was administered.

As is well-known, cetuximab (a human-murine chimeric immunoglobulin G1 monoclonal antibody), is the first FDA approved agent in clinic against EGFR. The schedule of cetuximab in combination with CCRT in LA NPC has demonstrated promising preliminary results [1215]. However, all of the studies reported an unexpectedly higher incidence of acneiform rash and grade 3/4 oral mucositis and dermatitis. Table 6 summarizes the observed adverse effects from these studies. Collectively, $85.8 \%$ $100 \%$ of patients experienced an acneiform rash, and grade $3 / 4$ rash, mucositis and dermatitis occurred in $10 \%-39.4 \%, 66.7 \%-87 \%$ and $18.2 \%-25 \%$ of patients, respectively, which were much more common than those in our study. We ascribe these favorable normal tissue toxicity results to the feature of nimotuzumab. Compared with cetuximab, the affinity constant of nimotuzumab is lower. Nimotuzumab requires bivalent binding for stable attachment, which means that it selectively binds cells that express moderate to high EGFR levels, thus sparing normal tissues where EGFR expression is low [16]. Most recently, by a retrospective analysis [23], Liu and colleagues also concluded that nimotuzumab could be safely combined with concurrent chemoradiation in the treatment of LA NPC.

The role of neoadjuvant or adjuvant chemotherapy remains ambiguous. The updated MAC-NPC metaanalysis confirmed that the addition of concomitant chemotherapy to radiotherapy significantly improves survival. However, this analysis does not completely answer the question whether there is a benefit of neoadjuvant or adjuvant chemotherapy in the concomitant setting [24]. There was no evidence of overall survival benefit observed with neoadjuvant or adjuvant chemotherapy. In addition, trends seen in specific trials showed that the additional treatment of neoadjuvant or adjuvant chemotherapy was poorly tolerated with increased toxic effects, and consequently, any possible benefit on survival may be offset $[25,26]$. In our study, one cycle of induction chemotherapy was administered during the time period of IMRT planning within 1 week prior to radiotherapy, with an intention of reducing tumor volume and preventing early disease progression, subsequent delivery of one cycle of concurrent chemotherapy was administered at intervals of 4 weeks after induction chemotherapy. Adjuvant chemotherapy with the same regimen of neoadjuvant setting was not mandatory but was planned for selected patients (those with good tolerance to concurrent chemoradiation and good response to induction chemotherapy), with the intention of reducing treatmentrelated toxicities. He and colleagues reported a similar treatment modality of chemoradiation plus cetuximab, preliminary survival data are encouraging compared with historic data [14].

In summary, our study showed that concurrent administration of chemoradiation and nimotuzumab was well-tolerated and showed encouraging clinical activities. With a median follow-up of 24.1 months, the estimated 2-year PFS and OS were $83.5 \%$ and $95.0 \%$, respectively, which appear promising in comparison with previous data $[27,28]$. However, our results were only for a short follow-up and a small sample size. Further randomized, controlled, larger sample clinical trials are warranted.

\section{MATERIALS AND METHODS}

\section{Patients}

Patients were eligible for enrollment if they were aged 18 years or older, with Eastern Cooperative Oncology Group (ECOG) performance status 2 or lower, all of them had histopathologically confirmed World Health Organization (WHO) type II or III at the primary site. All patients were staged according to the Union for International Cancer Control(UICC) 2010 staging system. Patients were eligible for inclusion if their tumors showed evidence of T3 or T4 stage and any $\mathrm{N}$ stage, M0 or N1-3 stage and any $\mathrm{T}$ stage, M0, as assessed by clinical examination, nasopharyngoscopy, MRI scan of the nasopharynx and neck, chest computed tomography, abdominal sonography, and bone scan. MRI was recommended for local staging. Other inclusion criteria were no evidence of metastatic disease, adequate hematological, liver, and renal function. Conditions for exclusion included prior radiotherapy or chemotherapy, EGFR inhibitors, other cancers, pregnancy, lactation, or clinically significant cardiopulmonary diseases. This study was approved by the Institutional Ethics Committee of the affiliated hospital of Jiangnan university. All patients signed written informed consent before participating in the study. 


\section{Treatment regimens}

\section{Radiotherapy}

Intensity-modulated radiotherapy was delivered at 70 Gy over 33 daily fractions to the planning target volume (PTV) of the primary tumor and metastatic cervical lymph nodes, 60Gy over 33 daily fractions to the subclinical disease around the primary tumor and upper neck, and 54 Gy to the lower neck and supraclavicular region in the absence of nodal involvement. For patients with persistent lesions at the nasopharynx or regional lymph nodes at the end of IMRT, a radiation boost was given(46Gy in 2-3 fractions).All patients were treated once daily, five fractions weekly. In order to delineate the target accurately, computerized optimization was used with the help of fusion of MRI and CT images in the treatment planning system. Dose constraints to the critical organs at risk (OAR) were applied according to the Radiation Therapy Oncology Group(RTOG) 0225 protocol.

\section{Chemotherapy and nimotuzumab therapy}

A flow chart depicting the drug delivery of chemotherapy and nimotuzumab is shown in Figure 1. According to the protocol, all patients were scheduled to receive one cycle of induction chemotherapy during the time period of IMRT planning within 1 week prior to radiotherapy, subsequent delivery of concurrent chemotherapy with the same regimen of neoadjuvant setting was administered at intervals of 4 weeks after induction chemotherapy. Four cycles of adjuvant chemotherapy (with the same regimen of neoadjuvant setting) was not mandatory but was planned for selected patients (those with good tolerance to concurrent chemoradiation and good response to induction chemotherapy), starting at 4 weeks after radiotherapy. Detailed inclusion criteria for adjuvant chemotherapy were Eastern Cooperative Oncology Group (ECOG) performance status 2 or lower, nonexistence of grade 3-4 radiotherapy-related skin and mucosal toxic effects, and adequate haematological, liver, and renal function at 4 weeks after radiotherapy. Chemotherapy regimens: docetaxel $75 \mathrm{mg} / \mathrm{m} 2$ on day 1 , nedaplatin $80 \mathrm{mg} / \mathrm{m} 2$ on day 2. Nimotuzumab was administered concomitantly with IMRT at a dose of $200 \mathrm{mg}$ weekly, commenced from the first day of radiotherapy. It was diluted in $250 \mathrm{~mL}$ saline and intravenously infused over 1 hour. Eight weeks of nimotuzumab was recommended but not mandatory.

\section{Patient evaluation and follow-up}

The assessment of tumor response used Response Evaluation Criteria for Solid Tumors(RECIST), which was performed 3months after the completion of radiotherapy with an MRI scan of the nasopharynx and neck. Evaluation of treatment-related toxicities began from the start of protocol therapy. Patients were monitored weekly during radiotherapy and before each chemotherapy cycle, with regard to hematologic and non-hematologic adverse effects. Systemic chemotherapy toxicities were assessed by the National Cancer Institute Common Toxicity Criteria (NCI CTCAE, version 3.0), whereas RT-related toxicities were graded according to the Acute and Late Radiation Morbidity Scoring Criteria of RTOG. Doses were modified in response to toxicities according to predefined guidelines.

Follow-up visits were at intervals of 3 months after radiotherapy for 2 years, 6 months for the next 3 years and annually thereafter. Assessments consisted of patient history, physical examination, MRI scan of the nasopharynx, chest computed tomography and abdominal sonography. Histological confirmation of locoregional recurrence and distant recurrence was encouraged.

\section{Study end points and statistics}

The main objective of the clinic trial was to evaluate the toxic effects and compliance of the combined modality treatment. Secondary endpoints included response rate to treatment, PFS and OS. OS was defined as the time from the date of enrollment to the date of death due to any cause or the date of lastfollow-up. PFS was the time between the date of enrollment and the first occurrence of locoregional or distant recurrence or the date of last follow-up. Locoregional recurrence was determined as the presence of recurrent disease at the nasopharynx and/or regional lymph nodes. Distant metastasis was defined as the presence of distant disease. Descriptive statistics [with 95\% confidence intervals (CIs) where applicable] were used to report the study end points and survival curve was estimated with the Kaplan-Meier method.

\section{ACKNOWLEDGMENTS}

This work was partially supported by grants from the National Natural Science Foundation of China (Nos. 31270897 and 81602793), and the medical scientific research project of Wuxi hospital management center (No. YGZXM152).

\section{CONFLICTS OF INTEREST}

The authors have no conflict of interest to declare.

\section{Author contributions}

Jian-feng Huang and Fu-zheng Zhang contributed to the trial design, study management and recruitment and treatment of the patients. Qin-zhou Zou, Le-yuan Zhou, Bo Yang and Jian-jun Chu were responsible for recruitment and treatment of the patients. Fen-ju Liu participated in the trial design, study management, data and toxicity review, review of the report, and final approval. C-M 
Charlie Ma contributed to the trial design, data analysis and interpretation, and review of the report. Jia-hua Yu were involved in trial design, writing of the protocol, and data management. Hao-wen Zhang were responsible for statistical analysis and interpretation, and toxicity and data review. Xiao-peng Yuan contributed significantly to the data analysis and report preparation. Guo-mei Tai participated in the trial design and writing of the report.

\section{REFERENCES}

1. Chan AT, Leung SF, Ngan RK, Teo PM, Lau WH, Kwan WH, Hui EP, Yiu HY, Yeo W, Cheung FY, Yu KH, Chiu KW, Chan DT, et al. Overall survival after concurrent cisplatin-radiotherapy compared with radiotherapy alone in locoregionally advanced nasopharyngeal carcinoma. J Natl Cancer Inst. 2005; 97: 536-9.

2. Wee J, Tan EH, Tai BC, Wong HB, Leong SS, Tan T, Chua ET, Yang E, Lee KM, Fong KW, Tan HS, Lee KS, Loong $\mathrm{S}$, et al. Randomized trial of radiotherapy versus concurrent chemoradiotherapy followed by adjuvant chemotherapy in patients with American Joint Committee on Cancer/International Union against cancer stage III and IV nasopharyngeal cancer of the endemic variety. J Clin Oncol. 2005; 23: 6730-8.

3. Lai SZ, Li WF, Chen L, Luo W, Chen YY, Liu LZ, Sun Y, Lin AH, Liu MZ and Ma J. How does intensitymodulated radiotherapy versus conventional twodimensional radiotherapy influence the treatment results in nasopharyngeal carcinoma patients? Int J Radiat Oncol Biol Phys. 2011; 80: 661-8.

4. Ng WT, Lee MC, Hung WM, Choi CW, Lee KC, Chan OS and Lee AW. Clinical outcomes and patterns of failure after intensity-modulated radiotherapy for nasopharyngeal carcinoma. Int J Radiat Oncol Biol Phys. 2011; 79: 420-8.

5. Sun X, Su S, Chen C, Han F, Zhao C, Xiao W, Deng $\mathrm{X}$, Huang S, Lin C and Lu T. Long-term outcomes of intensity-modulated radiotherapy for 868 patients with nasopharyngeal carcinoma: an analysis of survival and treatment toxicities. Radiother Oncol. 2014; 110: 398-403.

6. Ma BB, Poon TC, To KF, Zee B, Mo FK, Chan CM, Ho S, Teo PM, Johnson PJ and Chan AT. Prognostic significance of tumor angiogenesis, $\mathrm{Ki} 67, \mathrm{p} 53$ oncoprotein, epidermal growth factor receptor and HER2 receptor protein expression in undifferentiated nasopharyngeal carcinoma-a prospective study. Head Neck. 2003; 25: 864-72.

7. Sun W, Long G, Wang J, Mei Q, Liu D and Hu G. Prognostic role of epidermal growth factor receptor in nasopharyngeal carcinoma: a meta-analysis. Head Neck. 2014; 36: 1508-16.

8. Zhang P, Wu SK, Wang Y, Fan ZX, Li CR, Feng M, $\mathrm{Xu}$ P, Wang WD and Lang JY. p53, MDM2, eIF4E and EGFR expression in nasopharyngeal carcinoma and their correlation with clinicopathological characteristics and prognosis: A retrospective study. Oncol Lett. 2015; 9: 113-
118.

9. Bonner JA, Harari PM, Giralt J, Cohen RB, Jones CU, Sur RK, Raben D, Baselga J, Spencer SA, Zhu J, Youssoufian $\mathrm{H}$, Rowinsky EK and Ang KK. Radiotherapy plus cetuximab for locoregionally advanced head and neck cancer: 5-year survival data from a phase 3 randomised trial, and relation between cetuximab-induced rash and survival. Lancet Oncol. 2010; 11: 21-8.

10. Koutcher L, Sherman E, Fury M, Wolden S, Zhang Z, Mo Q, Stewart L, Schupak K, Gelblum D, Wong R, Kraus D, Shah J, Zelefsky M, et al. Concurrent cisplatin and radiation versus cetuximab and radiation for locally advanced headand-neck cancer. Int J Radiat Oncol Biol Phys. 2011; 81: 915-22.

11. Rowan K. Should cetuximab replace cisplatin in head and neck cancer? J Natl Cancer Inst. 2010; 102: 74-8.

12. Ma BB, Kam MK, Leung SF, Hui EP, King AD, Chan SL, Mo F, Loong H, Yu BK, Ahuja A and Chan AT. A phase II study of concurrent cetuximab-cisplatin and intensitymodulated radiotherapy in locoregionally advanced nasopharyngeal carcinoma. Ann Oncol. 2012; 23: 1287-92.

13. Niu $\mathrm{X}, \mathrm{Hu} \mathrm{C}$ and Kong L. Experience with combination of cetuximab plus intensity-modulated radiotherapy with or without chemotherapy for locoregionally advanced nasopharyngeal carcinoma. J Cancer Res Clin Oncol. 2013; 139: 1063-71.

14. He X, Xu J, Guo W, Jiang X, Wang X and Zong D. Cetuximab in combination with chemoradiation after induction chemotherapy of locoregionally advanced nasopharyngeal carcinoma: preliminary results. Future Oncol. 2013; 9: 1459-67.

15. Feng HX, Guo SP, Li GR, Zhong WH, Chen L, Huang LR and Qin HY. Toxicity of concurrent chemoradiotherapy with cetuximab for locoregionally advanced nasopharyngeal carcinoma. Med Oncol. 2014; 31: 170.

16. Ramakrishnan MS, Eswaraiah A, Crombet T, Piedra P, Saurez G, Iyer H and Arvind AS. Nimotuzumab, a promising therapeutic monoclonal for treatment of tumors of epithelial origin. MAbs. 2009; 1: 41-8.

17. Wolden SL, Chen WC, Pfister DG, Kraus DH, Berry SL and Zelefsky MJ. Intensity-modulated radiation therapy (IMRT) for nasopharynx cancer: update of the Memorial Sloan-Kettering experience. Int J Radiat Oncol Biol Phys. 2006; 64: 57-62.

18. Kam MK, Teo PM, Chau RM, Cheung KY, Choi PH, Kwan WH, Leung SF, Zee B and Chan AT. Treatment of nasopharyngeal carcinoma with intensity-modulated radiotherapy: the Hong Kong experience. Int J Radiat Oncol Biol Phys. 2004; 60: 1440-50.

19. $\mathrm{Xu} \mathrm{T}$, Zhu G, He X, Ying $\mathrm{H}$ and $\mathrm{Hu} \mathrm{C}$. A phase III randomized study comparing neoadjuvant chemotherapy with concurrent chemotherapy combined with radiotherapy for locoregionally advanced nasopharyngeal carcinoma: updated long-term survival outcomes. Oral Oncol. 2014; 
50: $71-6$.

20. Xu J, He X, Cheng K, Guo W, Bian X, Jiang X, Zhang $\mathrm{L}$ and Huang S. Concurrent chemoradiotherapy with nedaplatin plus paclitaxel or fluorouracil for locoregionally advanced nasopharyngeal carcinoma: Survival and toxicity. Head \& neck. 2014; 36: 1474-80.

21. Huang XD, Yi JL, Gao L, Xu GZ, Jin J, Yang WZ, Lu TX, Wu SX, Wu RR, Hu WH, Xie WC, Han F, Gao YH, et al. Multi-center phase II clinical trial of humanized anti-epidermal factor receptor monoclonal antibody h-R3 combined with radiotherapy for locoregionally advanced nasopharyngeal carcinoma. Zhonghua Zhong Liu Za Zhi. 2007; 29: 197-201.

22. Zhai RP, Ying HM, Kong FF, Du CR, Huang S, Zhou JJ and $\mathrm{Hu} \mathrm{CS}$. Experience with combination of nimotuzumab and intensity-modulated radiotherapy in patients with locoregionally advanced nasopharyngeal carcinoma. Onco Targets Ther. 2015; 8: 3383-90.

23. Liu ZG, Zhao Y, Tang J, Zhou YJ, Yang WJ, Qiu YF and Wang H. Nimotuzumab combined with concurrent chemoradiotherapy in locally advanced nasopharyngeal carcinoma: a retrospective analysis. Oncotarget. 2016; 7 : 24429-35. doi: 10.18632/oncotarget.8225.

24. Blanchard P, Lee A, Marguet S, Leclercq J, Ng WT, Ma J, Chan AT, Huang PY, Benhamou E, Zhu G, Chua DT, Chen Y, Mai HQ, et al. Chemotherapy and radiotherapy in nasopharyngeal carcinoma: an update of the MAC-NPC meta-analysis. The Lancet. Oncology. 2015; 16: 645-55.
25. Tan T, Lim WT, Fong KW, Cheah SL, Soong YL, Ang MK, Ng QS, Tan D, Ong WS, Tan SH, Yip C, Quah D, Soo KC, et al. Concurrent chemo-radiation with or without induction gemcitabine, Carboplatin, and Paclitaxel: a randomized, phase $2 / 3$ trial in locally advanced nasopharyngeal carcinoma. International journal of radiation oncology, biology, physics. 2015; 91: 952-60.

26. Lee AW, Tung SY, Chua DT, Ngan RK, Chappell R, Tung R, Siu L, Ng WT, Sze WK, Au GK, Law SC, O’Sullivan B, Yau TK, et al. Randomized trial of radiotherapy plus concurrent-adjuvant chemotherapy vs radiotherapy alone for regionally advanced nasopharyngeal carcinoma. Journal of the National Cancer Institute. 2010; 102: 1188-98.

27. Chan AT, Teo PM, Ngan RK, Leung TW, Lau WH, Zee B, Leung SF, Cheung FY, Yeo W, Yiu HH, Yu KH, Chiu KW, Chan DT, et al. Concurrent chemotherapy-radiotherapy compared with radiotherapy alone in locoregionally advanced nasopharyngeal carcinoma: progression-free survival analysis of a phase III randomized trial. Journal of clinical oncology. 2002; 20: 2038-44.

28. Lee AW, Lau WH, Tung SY, Chua DT, Chappell R, Xu L, Siu L, Sze WM, Leung TW, Sham JS, Ngan RK, Law SC, Yau TK, et al. Preliminary results of a randomized study on therapeutic gain by concurrent chemotherapy for regionallyadvanced nasopharyngeal carcinoma: NPC-9901 Trial by the Hong Kong Nasopharyngeal Cancer Study Group. J Clin Oncol. 2005; 23: 6966-75. 